ISSN 0001-6002/2003/45/3/120-125

Acta Médica Costarricense, $\odot 2003$

Colegio de Médicos y Cirujanos

\title{
Los frijoles (Phaseolus Vulgaris): Su aporte a la dieta del costarricense
}

\author{
Ligia Rodríguez-Castillo, Xinia E. Fernández-Rojas
}

\begin{abstract}
Resumen: Los frijoles constituyen un alimento básico en la dieta del costarricense. Sin embargo, la cantidad y frecuencia con que se consumen ha venido disminuyendo, sobretodo en la zona urbana. ${ }^{1}$

Esta tendencia resulta preocupante, ya que el frijol es fuente de proteínas, hierro vegetal, fibra, ácido fólico, tiamina, potasio, magnesio, y zinc (USDA, 2000) ${ }^{2}$. Su aporte a la dieta del costarricense, a pesar de la disminución en su consumo, es importante por su contenido de nutrientes y de fitoquímicos los cuales son de utilidad en la prevención y tratamiento de varias de las patologías que afectan a la población costarricense. ${ }^{3}$

La presente revisión, documenta los efectos positivos que algunos nutrientes y otros elementos que aporta el frijol tienen en la prevención y el control de algunas de las enfermedades crónicas que constituyen problemas de salud pública en el país. Se abordan de manera particular la fibra, el ácido fólico y el magnesio. Asimismo, se hace referencia a otras sustancias fitoquímicas, presentes en los frijoles, que constituyen elementos protectores de la salud y que hacen de los frijoles un "alimento funcional". Finalmente, se ofrecen recomendaciones prácticas orientadas a mejorar la digestibilidad de este alimento, de manera que se contribuya a mantener e incrementar su consumo a nivel de los hogares costarricenses.
\end{abstract}

Descriptores: frijoles (phaseus vulgaris), valor nutritivo, dieta, enfermedades crónicas, digestibilidad.

Recibido: 28 de febrero, 2003

Aceptado: 22 de julio, 2003

\section{Aporte nutricional de los frijoles a la dieta costarricense}

Los frijoles constituyen un alimento básico en la dieta del costarricense. De acuerdo con la Encuesta Nacional de Nutrición de $1996^{1}$ un $96.7 \%$ de los costarricenses consume frijoles. Sin embargo, la cantidad y frecuencia con que se consumen ha venido disminuyendo. En el periodo comprendido entre 1966 y 1996 el consumo promedio de frijol pasó de 57 $\mathrm{g}$ de frijoles/p/ día 4 , a un consumo de $31 \mathrm{~g} / \mathrm{p} /$ día en 1996. Dicho consumo es menor en la zona urbana $(28,1 \mathrm{~g} / \mathrm{p} /$ día $)$ que en la rural $(42,9 \mathrm{~g} / \mathrm{p} /$ día $){ }^{1}$

El frijol es fuente de proteínas, hierro vegetal, fibra, ácido fólico, tiamina, magnesio,potasio y zinc (USDA, 2000) ${ }^{2}$. En

Escuela de Nutrición, Universidad de Costa Rica

Correspondencia: Ligia Rodríguez Castillo, Escuela de Nutrición, Universidad de Costa Rica,Email: farodri@ racsa.co.cr la figura 1, puede observarse el aporte de energía y nutrientes de los frijoles a la dieta del costarricense. Dicho aporte es expresado como porcentaje de las recomendaciones dietéticas diarias (RDD), tomando como base el consumo reportado en la última Encuesta Nacional de Nutrición (1996), y en referencia a los requerimientos de adultos de ambos sexos ${ }^{5}$.

La figura 1 muestra a los frijoles como importantes contribuyentes a los requerimientos diarios de folatos, magnesio y fibra. En los niveles actuales de consumo, también es significativo el aporte de proteínas, tiamina, hierro y potasio. Cabe hacer notar que, a pesar de la significativa contribución de los frijoles, tanto los folatos como la fibra, siguen siendo deficientes en la población costarricense. ${ }^{6,7}$ Por lo tanto, los profesionales de la salud deben estimular al consumo frecuente de frijoles y otros alimentos fuente de fibra y folatos.

\section{Los frijoles contribuyen a prevenir enfermedades crónicas}

Las enfermedades cardiovasculares, la diabetes y el cáncer, constituyen serios problemas de morbimortalidad en el país ${ }^{10}$. Los frijoles contribuyen a la prevención y el tratamiento de estas patologías, tanto por su aporte de micronutrientes (particularmente ácido fólico y magnesio) como por su alto 


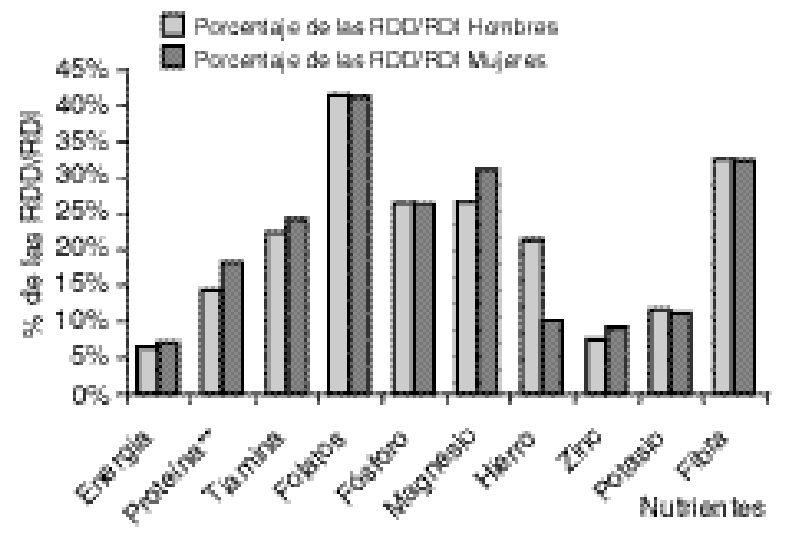

Figura 1. Aporte nutricional del consumo de frijoles a la dieta del costarricense. $1996^{\star}$

* Los datos se basan en la composición de frijol negro cocido (USDA, 2000.NBD \#16-015).

** Las RDD (Recomendaciones Dietéticas Diarias) se tomaron de: INCAP5; para potasio de las DRVS8;para fibra de Guías Alimentarias7; para folatos de $\mathrm{FAO} / \mathrm{WHO}$ y para proteína corresponde a $1 \mathrm{~g} / \mathrm{kg}$ de peso ideal /día.

contenido de fibra, aminoácidos azufrados, taninos, fitoestrógenos y aminoácidos no esenciales. Los efectos específicos del ácido fólico y de la fibra han sido ampliamente documentados ${ }^{11-16}$. El magnesio también ha sido identificado como un elemento involucrado en estos procesos, aunque su participación no ha sido completamente dilucidada ${ }^{17-19}$. Con respecto a otros elementos y a las interrelaciones bioquímicas que pueden potenciar estos efectos, hay trabajos de investigación que han abordado el alimento como un todo en vez de nutrientes específicos ${ }^{20-22}$.

Seguidamente se describen algunos de los hallazgos alrededor de la fibra, el ácido fólico, y el magnesio y sus correspondientes roles fisiológicos, que explican sus efectos positivos sobre la salud. Asimismo, se plantea la importancia de otros elementos presentes en los frijoles y del alimento en forma integral.

\section{Fibra}

La fibra comprende un grupo heterogéneo de polisacáridos tales como celulosa, hemicelulosa, pectina y de algunas otras sustancias que no corresponden al grupo de carbohidratos tales como la lignina, cuya característica genérica es que no pueden ser digeridos por el organismo humano.

Sin embargo, a través del tracto gastrointestinal pueden interactuar con diferentes elementos y captar agua. Este hecho, hace de la fibra un elemento muy importante en el proceso de la digestión, ya que limita y/o disminuye la velocidad de absorción de algunos nutrientes, y favorece el tránsito intestinal. Dadas estas características, la fibra permite una absorción más lenta de la glucosa, lo cual condiciona índices glicémicos moderados y, por lo tanto contribuye a con- trolar la hiperinsulinemia ${ }^{14,15}$. Esto tiene efectos benéficos tanto en la prevención como en el tratamiento de la Diabetes Mellitus.

Una dieta alta en fibra contribuye a mejorar el perfil lipídico, dado que disminuye la absorción intestinal de ácidos grasos y colesterol, tanto de la dieta como del colesterol reciclado proveniente de la bilis. Por otra parte, la fibra soluble libera, por efecto de fermentación, ácidos grasos de cadena corta, los cuales parecen ejercer un efecto inhibidor de la síntesis endógena de colesterol ${ }^{23,24}$. Todos esos efectos se refuerzan debido a que, en general, las dietas altas en fibra tienden a ser bajas en grasas y carbohidratos simples ${ }^{19}$.

De esta forma, los alimentos ricos en fibra dietética, tales como los frijoles, tienen el potencial de disminuir el riesgo de enfermedad cardiovascular desde diversas rutas: 1) por su efecto reductor del colesterol y los triglicéridos; 2) por su contribución al control glicémico, la Diabetes Mellitus y las complicaciones cardiovasculares asociadas; 3 ) por su aporte al mantenimiento de un peso adecuado (por la sensación de saciedad que provoca y por la composición de la dieta ya indicada). 14,15,19,23

Con respecto a cáncer, numerosos estudios tanto epidemiológicos como experimentales ${ }^{16,25,26,27}$ han demostrado una asociación inversa entre el consumo de fibra y el cáncer de colon. El efecto se explica porque acelera el tránsito intestinal, aumenta el volumen fecal y la frecuencia de defecaciones,así como porque influye en el metabolismo bacteriano, disminuyendo la formación y/o absorción de metabolitos cancerígenos. ${ }^{16,25,26}$ Por otra parte, Bruce, Wolever y Giacca ${ }^{27}$, reconociendo que la insulina y los triglicéridos pueden tender a incrementar el crecimiento de lesiones precursoras del cáncer de colon, proponen de nuevo, que el control de la hiperinsulinemia y la menor absorción de grasa, (ambos favorecidos por la fibra) ejercen un efecto protector sobre el cáncer de colon.

Dada la importancia de la fibra, el documento "Guías Alimentarias para Costa Rica" 7, recomienda un consumo de 30 g/p/día. Los costarricenses consumen una cantidad de fibra que está por debajo de esa meta. Diversos estudios ${ }^{28,29,30}$ han encontrado niveles de consumo en un rango de 11 a $20.3 \mathrm{~g}$ de fibra/p/día, con un promedio de $17 \mathrm{~g} / \mathrm{p} /$ día, en adultos costarricenses; lo cual indica la importancia de promover un mayor consumo de este elemento.

\section{Folatos}

Los folatos intervienen, como parte de un complejo enzimático en los procesos asociados con la reproducción celular. Así, tienen un efecto positivo sobre el mantenimiento de los tejidos, sobretodo de aquellos que requieren de un rápido recambio. Uno de los posibles mecanismos que explican este efecto es la intervención del folato en la metilación de compuestos tales como purinas y timidinas que, si no están disponibles, alteran la integridad y reparación del DNA ${ }^{11}$. Se 
plantea que estas alteraciones aumentan la carcinogénesis, al afectar la expresión de genes supresores de tumores y de proto-oncogenes. ${ }^{12}$ Esto podría explicar su contribución en la disminución del riesgo de desarrollar cáncer en determinados tejidos. Diversos estudios ${ }^{26,27}$ han demostrado una asociación positiva entre niveles altos de folatos y una menor incidencia de cáncer de colon.

Adicionalmente, la deficiencia de folatos provoca un incremento en los niveles de homocisteína, lo cual causa deterioros relacionados con la formación de placas de ateroma. ${ }^{13}$ Esto justifica el aporte de los folatos en la prevención de la enfermedad cardiovascular.

En respuesta a todos estos hallazgos, el Grupo de Expertos $\mathrm{FAO} / \mathrm{WHO}$ en requerimientos de vitaminas y minerales del ser humano, en un reporte preliminar, revisado en julio del 2000, recomienda duplicar la RDD de folatos, de 200 a 400 $\mathrm{mcg} / \mathrm{p} /$ día. (FAO/WHO, 1998).9 Los frijoles aportan a la dieta costarricense (con una media de consumo de 31g/p/día; según la Encuesta de 1996, ${ }^{1}$ ) un 32\% de esa recomendación. Mayores ingestas de folatos podrían ser conseguidas, sin suplementación, incrementando el consumo de frijoles.

\section{Magnesio}

El magnesio tiene un papel importante en al menos 300 reacciones enzimáticas esenciales. Posiblemente, debido a esos diversos roles, existen aún contradicciones con respecto a su posible contribución en la prevención y tratamiento de algunas enfermedades ${ }^{18}$. No obstante, es claro que magnesio forma parte de numerosas enzimas, algunas de las cuales constituyen potentes antioxidantes en la cadena respiratoria que lleva a la generación de ATP y agua a nivel de la mitocondria. Además, funciona en la activación de aminoácidos y en la síntesis y degradación del DNA ${ }^{19}$. Estos roles lo identifican como un nutriente importante con respecto a la prevención del cáncer. Por otra parte, el magnesio parece ejercer un efecto protector contra la hipertensión y, consecuentemente, los problemas cardiacos ${ }^{17}$.

\section{Los frijoles son fuente de fitoquímicos}

Además de los efectos ya discutidos de la fibra, los folatos y el magnesio en la prevención de varias de las enfermedades crónicas y degenerativas que afectan a la población costarricense, es necesario también señalar que otras sustancias fitoquímicas presentes en los frijoles, tales como: fitoestrógenos, taninos, aminoácidos sulfurados y un conjunto amplio de aminoácidos no esenciales, también han sido identificados como elementos protectores contra algunos de los factores que estimulan el crecimiento de los tumores. ${ }^{20,31}$

Slavin ${ }^{21}$, al resumir el efecto protector de los granos enteros (leguminosas y cereales integrales) sobre varios tipos de cáncer (gástrico, colon, pecho y próstata), indica los posibles mecanismos: 1) son una fuente concentrada de fibra; 2) son ricos en antioxidantes, incluyendo minerales como el magnesio; 3 ) son ricos en folatos (las leguminosas); 4) son una fuen- te de fitoestrógenos que tienen efectos hormonales relacionados con la prevención del cáncer de mama y de próstata y 5) median la respuesta insulínica, lo cual se ha planteado que protege contra el cáncer de colon y el cáncer de mama. Es por eso que las leguminosas, han sido catalogadas como un "alimento funcional"; ${ }^{32}$ es decir un alimento que además de nutrientes, aporta otras sustancias que tienen un efecto benéfico sobre la salud. ${ }^{33,34}$

Cuando se evalúa el efecto de un alimento sobre la salud, una consideración importante es el sinergismo que puede existir entre sus diferentes elementos constitutivos (nutrientes y nonutrientes), lo cual puede potenciar o reducir su efecto benéfico ${ }^{32}$. Por esta razón, la comunidad científica ha abordado el estudio no solo de los efectos de componentes aislados, sino también de diferentes alimentos en forma integral.

Con respecto a frijoles, un estudio epidemiológico de Correa ${ }^{35}$, encontró una correlación inversa, altamente significativa entre el consumo de frijoles y la mortalidad ajustada por edad de cáncer de mama $(\mathrm{r}=-0.70)$; cáncer de próstata $(\mathrm{r}$ $=-0.66)$, y cáncer de colon $(r=-0.68)$. Kolonel et al. ${ }^{36}$ reportaron también una tendencia significativa inversa entre el consumo de leguminosas (excluyendo alimentos de soya) y la incidencia de cáncer de próstata. Hangen y Benninck ${ }^{22}$ y Hughes et $\mathrm{al}^{37}$ determinaron, utilizando un modelo animal, que la alimentación con frijoles inhibió la incidencia de tumores químicamente inducidos en el colon.

Considerando la evidencia, diversos autores y entidades reconocidas, recomiendan dietas con una alta proporción de alimentos de origen vegetal, entre las que destacan los vegetales y las frutas. 26,38-40. Con respecto a leguminosas, el Fondo Mundial para la Investigación en Cáncer (World Cancer Research Fund) y el Instituto Americano para la Investigación en Cáncer (The American Institute for Cancer Research ${ }^{38}$ reconocen como biológicamente plausible un efecto positivo de las leguminosas en la prevención del cáncer, y recomiendan dar prioridad a estudios en que se determine esta relación. La Asociación Americana del Corazón (American Heart Association) presentó recientemente una nueva guía dietética, que hace énfasis en los alimentos más que en los nutrientes y recomienda al menos seis porciones diarias de leguminosas y granos integrales. ${ }^{41}$

\section{Los problemas de digestibilidad de los frijoles y como resolverlos}

A pesar de todas las ventajas señaladas, es necesario también reconocer los problemas de digestibilidad y flatulencia que, algunos individuos presentan, al consumir frijoles y que podrían limitar su consumo.

Estos problemas son ocasionados por oligosacáridos no digeribles (rafinosa y estaquiosa) que son fermentados en el colon por bacterias y producen hidrógeno, metano y dióxido de carbono. ${ }^{42}$ Sin embargo, es necesario señalar que, esos mismos oligasacáridos, favorecen el crecimiento de bifidobacterias que se ha demostrado que disminuyen el riesgo de cáncer de colon $^{43}$. 


\begin{abstract}
Para preservar el valor nutritivo
1. Deje los frijoles en remojo por lo menos por 8 horas.45 Dejarlos la noche anterior del día de la cocción es una práctica sencilla. Para mayor preservación de nutrientes no elimine el agua de remojo.
\end{abstract}

Cuadro 1

Recomendaciones prácticas para preservar el valor nutritivo de los frijoles

y evitar los problemas de flatulencia

Para evitar flatulencia y

mejorar la digestión

2. Cocine preferiblemente en "olla mágica”. La cocción rápida contribuye a conservar nutrientes. 46

3. Cocine sin sal, hasta máxima suavidad o al gusto del consumidor. Agregue la sal y/o otros condimentos hasta el final de la cocción.

4. Ya cocinados, separe los frijoles en porciones del tamaño que usted considere son suficientes para 1 día. 45 Deje enfriar a temperatura ambiente y coloque en el refrigerador los que necesita para dos días. El resto congélelos en envases cerrados.

5. Descongele los frijoles del día y caliente solamente, cuando vaya a consumirlos. Evite recalentar los frijoles una y otra vez.

6. Estas prácticas preservan el valor nutritivo del frijol, especialmente del ácido fólico y la tiamina que se pierden por exposición prolongada al calor.46,47.
1. Mastique muy bien. La digestión se inicia en la boca.

2. Introduzca los frijoles gradualmente en su dieta, especialmente si tiene mucho tiempo de no consumirlos.

3. Si el frijol entero causa problemas, aun después de haber sido remojado y estar bien cocinado, trate consumiéndolos molidos o licuados.

4. Revise la forma de preparación y pruebe eliminando salsas, cominos, pimienta y grasa. Esto reduce agentes que pueden ser irritantes del tracto gastrointestinal. 45

5. Pruebe también preparando frijoles rojos en vez de negros. Los primeros han demostrado ser más digeribles. 48

6. Solo si los problemas de flatulencia o disconformidad intestinal persisten, elimine el agua de remojo y agregue agua limpia para cocinar.49
1. Para la alimentación infantil, utilice el frijol entero, molido o licuado. El caldo de frijol, sin frijoles, no es una adecuada fuente de nutrientes para este grupo de edad.50

2. Pruebe nuevas alternativas y recetas para la preparación de frijoles para la familia.45
Otros factores antinutricionales identificados son las antitripsinas (proteínas que inhiben la acción de la tripsina), y algunos glicósidos. Estos son termolábiles los primeros y volátiles los segundos, lo cual implica que la cocción usual elimina su actividad ${ }^{44}$.

Los problemas de digestibilidad de los frijoles pueden ser superados, en la mayor parte de los casos, mediante procedimientos adecuados de preparación y consumo. En Cuadro 1 , se presenta un resumen de algunas recomendaciones para evitar estos problemas y para preservar el potencial nutritivo de este alimento.

En conclusión, al promover el consumo de frijoles, se podría incrementar el aporte de nutrientes, fibra y fitoquímicos importantes que contiene este alimento, lo que contribuiría al mejoramiento de la alimentación y a la prevención de algunos de los problemas de salud que actualmente afectan a la población costarricense. Además, se contribuiría con el rescate de un alimento que forma parte de nuestra cultura y seguridad alimentaria .

Es por eso que la Escuela de Nutrición ha desarrollado el proyecto "Campaña educativa para la promoción del consumo de frijoles en una comunidad urbana"; el cual ha generado diversas publicaciones, materiales educativos s, $^{3,55,51,52,53,54}$ y experiencias de utilidad para la promoción del consumo de este alimento de interés nacional. 


\section{Agradecimientos y colaboraciones}

Este artículo se elabora dentro del marco del Programa "Bean Cowpea Collaborative Research Program”, fondo DAN-GSS-860008-88 AID. Dicho programa apoya al proyecto macro "Digestibilidad del Frijol Común", a cargo del Centro Nacional de Investigación en Tecnología de Alimentos (CITA) de la UCR. Como parte de ese proyecto macro, la Es cuela de Nutrición de la Universidad de Costa Rica, ha venido trabajando en la transferencia tecnológica y educación nutricional al consumidor.

\section{Abstract}

Beans (Phaseolus vulgaris ) are an important part of the Costa Rican diet. Nevertheless, the amount and the frequency of its consumption has been decreasing over time, especially in the urban areas.

This tendency is a concern to those involved in health and nutrition since beans are a source of protein, non-heme iron, fiber, folic acid, thiamin,potassium , magnesium and zinc. The nutritional contribution of beans to the Costa Rican diet is important in spite of the reduction in consumption. Beans are important because of their nutrient content and the presence of phytochemicals that have been recognized as part of the prevention and treatment of health problems affecting the Costa Rican population.

This review documents the positive effects of some of the nutrients and elements present in beans related to the prevention and control of some chronic diseases of importance in public health. It includes information on fiber, folic acid and magnesium. Additionally, it considers the presence of phytochemicals that make beans a "functional food".

Finally, some practical recommendations are offered in order to improve digestibility. Thus, our purpose is to educate the Costa Rican population to maintain and to increase the consumption of beans.

\section{Referencias}

1. Ministerio de Salud. Encuesta Nacional de Nutrición Fascículo 3:Consumo Aparente. San José, Costa Rica:Ministerio de Salud; 1996.

2. U.S. Department of Agriculture. Base de datos sobre composición de alimentos. http:// www.nal.usda/fnic; 2000

3. Campos E, Fernández A,Sánchez I,Sancho T, y Villalobos C. Diagnóstico para el diseño de una campaña educativa tendiente a incrementar el consumo de frijol en una comunidad urbana de clase media de San José. Seminario de Licenciatura. San José, Costa Rica: Universidad de Costa Rica; 1999.

4. INCAP/ICNND/ Ministerio de Salubridad Pública de Costa Rica. Evaluación Nutricional de la Población de Centroamérica y Panamá:Costa Rica. Publicación INCAP-V-28. Guatemala, Guatemala: INCAP; 1969.

5. Torún B, Menchú M, Elías L. Recomendaciones Dietéticas Diarias del INCAP:Edición XLV Aniversario. Publicación INCAP ME/057. Guatemala: INCAP; 1996
6. Ministerio de Salud/INCIENSA. Encuesta Nacional de Nutrición Fascículo 2: Micronutrientes. San José, Costa Rica: Ministerio de Salud; 1996.

7. Muñoz L, Murillo S; ed. Guías de Alimentación: Lineamientos metodológicos y criterios técnicos. San José, Costa Rica: INCAP/OPSUCR; 1995.

8. Whitmire, S.J. Water, Electrolyte and Acid-base Balance. En: Mahan, $\mathrm{K}$ and Escott-Stump. Krause's Food, Nutrition and Dietherapy. 10th Ed. Philadelphia, USA:Saunders Pub; 2000. p. 159

9. FAO/WHO (1998). Preliminary Report on Recommended Intakes of the Joint FAO/WHO Expert Consultation Group on Human Vitamin and Mineral Requirements. Reviewed July 2000. http://www.fao.org.

10. Sáenz, L. Evolution of an Epidemiological Profile. En: Muñoz C y Scrimshaw N, ed. The Nutrition and Health Transition of Democratic Costa Rica. Boston, USA: International Foundation for Developing Countries; 1995. p. 119-143.

11. Suárez, M.P. Acido Fólico: Nutriente Redescubierto. Acta Médica Costarricence 2003; 45(1):5-9

12. Sang-Woon, ,Choi , Mason, J. Folate and carcinogenesis: and integrated scheme. J.Nutr 2002; 132(2):129-133.

13. Beresford S, Boushey C. Homocysteine, Folic Acid and Cardiovascular Disease Risk. En: Bendich A y Deckelbaum R,ed. Preventive Nutrition. New Jersey, USA:Humana Press; 1997. p. 193-224

14. Ludwig D, Pereira M,Kroenke C,Hilner J, Van-Horn L, Slattery M et al. Dietary fiber, weight gain, and cardiovascular disease risk factors in young adults. J Am Med Assoc 1999; 282: 1539 - 1546.

15. Chandalia M, Gar A, Lutjohann D, Von Bergmahn K, Gundry S, Brirkelley L. Beneficial Effects of Dietary Fiber intake in Patients with Type 2 Diabetes Mellitus. New Eng J Med 2000; 342 (19):1440-1441.

16. Reddy B. Prevention of colon carcinogenesis by components of dietary fiber. Anticancer Res 1999; 19(5A): 3681-3.

17. Polisso G, Barbagallo M. Hypertension, diabetes mellitus and insulin resistance: the role of intracellular magnesium. Am J Hyperten 1997; 10: 346-355

18. Laurant P, Touyz R. Physiological and Pathophysiological Role of Magnesium in the Cardiovascular System: Implications in Hypertension. J Hipertens 2000; 18 (9): 1171-1191.

19. Whitney E, Rolfes S. Understanding Normal and Clinical Nutrition. $8^{\text {a }}$ Ed. USA: Wadsworth Publishing Company; 1999.

20. Messina M. Legumes and soybeans: overview of their nutritional profiles and health effects. Am J Cl Nut 1995; 70 (3 Suppl.) 439S-450S.

21. Slavin J. Mechanisms for the Impact of Whole Grain Foods on Cancer Risk. J Am Coll Nutr 2000; 19 (3 suppl):300S-307S.

22. Hangen L y Bennink M.R. Consumption of Phaseolus vulgaris (Black Beans or Navy beans) Reduces Colon Cancer in Rats. FASEB J 2001; 15:A61.

23. Bourges-Rodríguez H. Aterosclerosis. En: Casanueva E, Kaufer-Horwitz A, Pérez-Lizaur M y Arroyo P, ed. Nutriología Médica. Publicación de la Fundación Mexicana para la Salud. México D.F.: Editorial Médica Panamericana S.A. 1996. p. 232-254.

24. Mahan L,Escott-Stump S. Nutrición y Dietoterapia de Krause. $10^{\text {a }}$ Ed. México: MacGraw-Hill Interamericana; 2000.

25. Grasten S, Juntunnen K, Poutanen K, Gylling H, Miettinen T, Mykkanen $\mathrm{H}$. Rye bread improves bowel function and decreases the concentrations of some compounds that are putative colon cancer risk markers in middle-aged women and men. J Nutr 2000; 130(9):2215-21.

26. Bostic R. Diet and Nutrition in the Etiolo gy and Primary Prevention of Colon Cancer. En: Bendich A y Deckelbaum R, ed. Preventive Nutrition. New Jersey, USA: Humana Press,1997. p. 57-95.

27. Bruce W, Wolever T, Giacca A. Mechanisms linking diet and colorectal cancer:the possible role of insulin resistance. Nutr Cancer 2000; 37 (1): $19-26$. 
28. Arauz A, Monge R, Muñoz L, Rojas M. La dieta como factor de riesgo de la enfermedad cardiovascular en habitantes del área metropolitana. San José, Costa Rica. Arch Latinoam Nutr 1991; 41: 350-362.

29. Quintana R. Características de la dieta en relación con enfermedad cardiovascular en el Distrito la Fortuna de San Carlos. Tesis de Licenciatura. San José,Costa Rica: Universidad de Costa Rica; 1994.

30. INCAP/OPS/COOPESAIN. Diagnóstico de factores de riesgo cardiovascular en el cantón de Tibás. San José,Costa Rica:EBAIS B1; 1995.

31. Mc Carty, M. Vegan protein may reduce risk of cancer, obesity and cardiovascular disease by promoting increased glucagon activity. Med Hypothes 1999; 53(6): 459-85.

32. Sedó, P. Alimentos Funcionales:análisis general acerca de las características químico-nutricionales, desarrollo industrial y legislación alimentaria. Rev. Costarricense de Salud Pública 2001; 10 (18 y19) 3439.

33. Sedó P. El mercado de alimentos funcionales y los nuevos retos para la educación alimentaria nutricional. Rev Costarricense de Salud Pública 2002; 11(20):18-25.

34. ADA. Functional foods-Position of ADA. J Am Diet Assoc. 1999; (99): 1278-1285.

35. Correa P. Epidemiological cor relations between diet and cancer frequency. Cancer Res 1981; 41:3685-90

36. Kolonel L, Hankin J, Whittemore A, Wu A, Gallagher R, Wilkens L et al. Vegetables, fruits,legumes and prostate cancer: a multiethnic casecontrol study. Cancer Epidemiol Biomark Prev 2000; 9:795-804.

37. Hughes J, Ganthavorn C, Wilson L, Sander S. Dry beans inhibit Asoxymethane-induced colon carcinogenesis in F344 rats. J Nutr 1997; 127 (12): 2328-33.

38. World Cancer Research Fund and American Institute for Cancer Research. Food, Nutrition and the Prevention of Cancer:A Global Perspective. USA: World Cancer Research Fund/American Institute for Cancer Research; 1997.

39. Ji B, Chow W, Yang G, McLaughlin J, Zheng W, Shu X, et al. Dietary Habits and Stomach Cancer in Shanghai,China. Int J Cancer 1998; 76 (5):659-64.

40. Cornee J, Pobel D, Riboli E, Guyander M, Hemon B. A Case-control Study of Gastric Cancer and Nutritional Factors in Marseille, France. Eur J Epidemiol 1995; 11(1): 55-56.

41. Hopkins,J. US Heart Doctors Recommend New Dietary Guidelines. Br Med J 2000; 321:918.

42. Messina M. Legumes and soybeans:Overview of their nutritional profiles and Health Effects. Am J Cl Nut 1999; (Suppl) 70: 439S-50S.

43. Sanders M. Probiotics:a publication of the Institute of Food Technologists Expert Panel on Food Safety and Nutrition. Food technology 1999; 53 (11): 67-76.

44. Sánchez L, Sedó P. Manual de Laboratorio Curso Alimentos 1 (NU1006). San José, Costa Rica: Universidad de Costa Rica, Facultad de Medicina, Escuela de Nutrición; 1998.

45. Dumani M, Rodríguez S. De Todo con Frijoles. San José, Costa Rica: Universidad de Costa Rica, Escuela de Nutrición, CITA-Programa Bean Cowpea CRSP-AID; 2000.

46. Fox B, Cameron A. Ciencia de los Alimentos, Nutrición y Salud. México: Editorial Limusa; 1999.

47. Augustin J, Beck A, Kalbfleish N, Kagel L,Mattehews R. Variation in the vitamin and mineral content of raw and cooked commercial Phaseous vulgaris classes. J Food Science 1981; 46:1701-06.

48. Poletti R, Torelli G. Evaluación de características tecnológicas y nutricionales de 12 variedades negras y 12 variedades rojas de frijol (Phaseolus vulgaris) como complemento de los criterios de selección. Proyecto de licenciatura. San José, Costa Rica: Universidad de Costa Ri ca; 1985
49. Víquez F. Determinación de los principales polisacáridos no almidonosos causantes de flatulencia en el frijol común. Efecto de la adición de una pectinasa y una celulasa sobre su digestibilidad y aceptabilidad. Tesis de Magister Scientae. San José,Costa Rica: Universidad de Costa Rica; 1999.

50. Ulate E. Grasas y Deficiencias Nutricionales. En: Fernández, X. ed. Memorias Seminario Grasas y Alimentación Humana. San José, Costa Rica:Litografía Raval, 1994. p. 17-31.

51. Martínez, T; Murillo,A; Rodríguez, S y Páez, P. (2000). Elaboración de los Contenidos de los Mensajes de una Campaña Educativa para Aumentar el Consumo de Frijoles en una Comunidad Urbana de Clase Media. Seminario de Licenciatura. San José, Costa Rica: Universidad de Costa Rica.

52. Rodríguez,L. y Dumani,M. (2000 a). Diagnóstico para una Campaña Educativa para la Promoción del Consumo de Frijoles. En: Libro de Resúmenes de Trabajos Libres del XII Congreso Latinoamericano de Nutrición; SLAN 2000. Buenos Aires,Argentina:Secretaría Científica CESNI.

53. Rodríguez,L y Dumani M. (2000 b). Campaña Educativa con Respecto al Consumo de Frijoles. En: Memorias del IV Taller Anual de Resultados de Investigación y Transferencia de Tecnología PITTA-Frijol. Ed. Por Revista Agronomía Mesoamericana. San José, Costa Rica: Profrijol.

54. Rodríguez,L, Dumani,M, Páez, P e Invancovich, C. (2002). Informe Julio del 2002 del Proyecto "Campaña Educativa para promocionar el Consumo de Frijoles". San José,Costa Rica:Universidad de Costa Rica, Facultad de Medicina, Escuela de Nutrición. Documento impreso. 